\title{
Statistical Modelling of the Effect of Alcohol and Sound Intensity on Response to Fire Alarms
}

\author{
ABRAHAM HASOFER, IAN THOMAS, DOROTHY BRUCK, and \\ MICHELLE BALL \\ CESARE and School of Psychology \\ Victoria University \\ P.O. Box 14428 \\ MCMC Melbourne, Victoria 8001 Australia
}

\begin{abstract}
A stochastic model for analysing the response of sleeping subjects to sound stimuli whose intensity varies with time is developed, generalizing a model developed in [1] for analysing the response of sleeping subjects to sound stimuli of constant intensity. The model is used to analyse the results of an experiment carried out by Ball and Bruck [2] to compare the response time of sleeping subjects to three different auditory stimuli. The sound intensity increased steadily with time and the young adult subjects (seven males and seven females) were tested when sober and with blood alcohol levels of 0.05 and 0.08 . The analysis revealed that alcohol had a very significant effect in slowing down the response of all subjects. It also revealed that females responded faster than males at all alcohol levels. The great advantage of using the stochastic model is that it permits the estimation of the probability that the response time will exceed high values that may put the sleeping occupant at a severe risk of death or injury in a fire.
\end{abstract}

KEYWORDS: human behaviour, auditory fire cues, response time, influence of sound type, influence of gender, influence of alcohol, stochastic modelling

\section{INTRODUCTION}

In two previous papers $[1,3]$ the authors have introduced a random walk model to analyse the length of time to response of sleeping subjects exposed to fire cues. In the first paper, two experiments were analysed. In the first one, sleeping young adults were exposed to a smoke detector alarm received at $60 \mathrm{dBA}$. In the second one, children were exposed to an alarm that was received at $89 \mathrm{dBA}$. In the second paper, two further experiments were analysed. In the first, adults aged 25-55 years were exposed to two auditory cues (a crackling noise and a "shuffling" noise),and a visual cue (a flickering light). The intensity of the auditory cues was about $45 \mathrm{dBA}$ at the pillow, while the intensity of the light cue was about 5 lux. In the second experiment, young adults were exposed to an olfactory cue mimicking fire smoke. A dispenser emitted puffs of a mixture of guaiacol in an ethanol base, resulting in a concentration at the pillow of about 2 parts per million.

\section{The Random Walk Model}

It was pointed out in [3] that one of the most important aims of the study of the response time to fire cues was to estimate the high quantiles of the distribution of the response time, since it is those who respond the most slowly who are the most at risk. However, the raw experimental results based on a comparatively small number of observations cannot by themselves be extrapolated to the levels that are infrequently reached. The only alternative is to develop a theoretical model for the response to fire cues and fit it to the experimental data. The model that was developed to analyse the experiments just described is called a memory retrieval theory because it considers an item recognition 
task as testing a single probe item against a group of items in memory which has been designated as the memory search set. The retrieval process consists of comparing a collection of features of each element of the memory search set with the probe item. In the application of the model to the response of subjects to a fire cue the probe item is identified with the stimulus induced in the sleeping subject by the cue. This stimulus is compared with the items of the memory search set. Comparison of a probe to a memoryset item proceeds by the gradual accumulation of evidence, that is, information representing the goodness of match, over time. It is easiest to conceptualize the comparison process as a feature-matching process in which probe and memory-set item features are matched one by one. A count is kept of the combined sum of the number of feature matches and nonmatches, so that for a feature match, a counter is incremented, and for a feature nonmatch, the counter is decremented. The counter begins at some starting value $Z$, and if a total of $A$ counts are reached, the probe is declared to match the memory-set item (i.e., $A$ - $Z$ has more feature matches than nonmatches). But if a total of zero counts are reached, an item nonmatch is declared. The response is thus modelled by a random walk bounded from above and below. The lower boundary is taken to be 0 . The upper boundary is denoted by $A$ and the starting value by $Z$. The process ends when either of the boundaries is reached. If the lower boundary is reached first the subject dismisses the stimulus and continues to sleep. If, on the other hand, the upper boundary is reached first the subject wakes up. Thus, the upper boundary $A$ can be thought of as a threshold of sensitivity to the stimulus. In the experiments analysed in both papers the intensity of the stimulus was intended to remain stable. On that account, the upper threshold was assumed to be fixed. The random walk is then described by four parameters:

1. $Z$, the starting value,

2. A, the wake up threshold,

3. the probability $p$ of a match between the stimulus and an item of the memory set, resulting in an upward jump of one unit at each step. A non-match, that occurs with probability 1-p, results in a downward jump of one unit. The parameter $p$ will be referred to in the rest of the paper as the recognition probability.

4. the length $d$ of one step.

Once a value for $A$ (or $Z$ ) is decided upon, the other three parameters of the random walk can be estimated from the data.

Choosing a value for $A$ involves a compromise between two conflicting considerations. On the one hand, choosing $A$ (or $Z$ ) too small would make the random walk too coarse. On the other hand, making $A$ (or $Z$ ) too large would vastly increase the amount of computation required without achieving a more significant fit. In [1] $A$ was taken to be 100 , and in [3] $Z$ was taken to be 50 .

\section{THE EFFECT OF SOUND INTENSITY AND ALCOHOL: METHODOLOGY}

In 2003 an experiment was carried out by Ball and Bruck [2] to compare the response time of sleeping subjects to three different auditory stimuli, as well as to determine the effect of sex and alcohol consumption on responsiveness. Fourteen young adults (7 males, 7 females) were recruited. The ages of the participants ranged from 18 to 25 years. All subjects reported that they felt themselves to sleep more deeply than average. 
When the participant was confirmed to be in stage 4 sleep for at least 90 seconds a computer program was started. The computer was instructed to play the selected sound for a period of 30 seconds, beginning at $35 \mathrm{dBA}$. The intensity of the sound was then increased to $40 \mathrm{dBA}$ for a further 30 seconds. The incremental pattern was continued until $95 \mathrm{dBA}$ or until the participant awoke and pressed a button at their bedside, whichever occurred first. The time dependence of the sound intensity is illustrated in Fig. 2. If the participant did not respond before 30 seconds at $95 \mathrm{dBA}$, the sound continued for a period of 3 minutes. The entire process from start to finish took 9.5 minutes. Participants who slept through the signal were coded as awakening after 10 minutes. As far as the sound intensity level was concerned, responses in the first 30 second period at $95 \mathrm{dBA}$ was recorded as $95 \mathrm{dBA}$, responses in the second period of 30 seconds at $95 \mathrm{dBA}$ were recorded as $96 \mathrm{dBA}$, and so on, up to $101 \mathrm{dBA}$ for awakening in the final (seventh) period at $95 \mathrm{dBA}$. Non-awakening was recorded as $105 \mathrm{dBA}$ (10 dBA higher than the real maximum sound level).

Participants were tested on 3 non-consecutive nights in three different conditions: no alcohol, 0.05 blood alcohol content and 0.08 blood alcohol content. The order of the latter two was counterbalanced.

The response time analysed in this paper is the time until the subject presses a button "Behavioural response". Awakening time was also assessed by means of an electroencephalogram (EEG), but these times are not reported in this paper as data analysis was not completed.

Three sounds were used:

1. Female Voice: This signal consisted of a female actor's voice that warned of danger due to fire in an emotional tone and said that the person must wake up and investigate. There was a core sound of 10 seconds duration, repeated 3 times in 30 seconds.

2. Australian Standard Alarm ("ASA”): This alarm signal was the modulating high frequency beeping sound that is used in residential smoke alarms in Australia.

3. Temporal-Three Evacuation Signal ("T3 Alarm”): A lower frequency alarm signal that sounds the Temporal-Three pattern as laid down in International Standard 8201 [4].

\section{EXPERIMENTAL RESULTS}

Table 1 lists the mean and standard deviation of the behavioural response time (in seconds) to each of the three sounds and each of the three alcohol levels, for males and females.

While the observed means are reasonably reliable, there are not enough data to ensure the reliability of the observed standard deviations. However, a plot of the standard deviations against the means (Fig. 1) shows that the standard deviations are roughly proportional to the means. Regression of the standard deviation on the mean (with zero intercept) shows that the ratio of the standard deviation to the mean (i.e., the "coefficient of variation") can be taken to be approximately constant at 0.49 . Analysis of variance confirms that the proportionality is highly significant. In the development of the model in the next section the coefficient of variation is taken to be constant with the value 0.49 just derived. 
Table 1. Mean and standard deviation of behavioural response time (male and female).

\begin{tabular}{|l|l|l|l|l|l|}
\hline Sound & $\begin{array}{c}\text { Alcohol } \\
\text { level }\end{array}$ & $\begin{array}{c}\text { Male } \\
\text { mean }\end{array}$ & $\begin{array}{c}\text { Male } \\
\text { standard } \\
\text { deviation }\end{array}$ & $\begin{array}{c}\text { Female } \\
\text { mean }\end{array}$ & $\begin{array}{c}\text { Female } \\
\text { standard } \\
\text { deviation }\end{array}$ \\
\hline $\begin{array}{l}\text { Female } \\
\text { voice }\end{array}$ & Sober & 168.43 & 91.95 & 119.43 & 80.55 \\
& 0,05 & 421.71 & 216.71 & 201.43 & 116.92 \\
& 0.08 & 369.71 & 161.95 & 227.14 & 189.16 \\
\hline ASA & Sober & 219.71 & 70.15 & 246.00 & 190.57 \\
& 0,05 & 385.43 & 124.81 & 246.14 & 108.77 \\
& 0.08 & 427.57 & 137.38 & 270.14 & 157.00 \\
\hline T3 & Sober & 174.57 & 88.43 & 125.43 & 82.35 \\
& 0,05 & 341.71 & 173.71 & 196.00 & 106.05 \\
& 0.08 & 425.71 & 166.81 & 195.00 & 187.25 \\
\hline
\end{tabular}

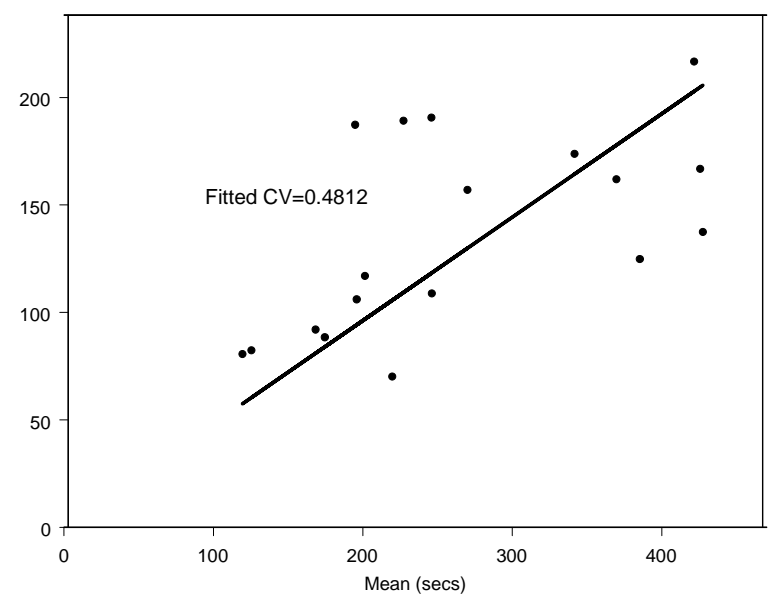

Fig. 1. Regression of observed standard deviation on observed mean.

(Regression line equation: standard deviation $=0.4812 \times$ mean $)$.

\section{BASIC MODELLING ASSUMPTIONS}

The experiment analysed in this paper differs from those analysed in [1] and [2] in a basic respect: while in the two papers just cited the intensity of the stimulus remained constant throughout the experiment, here the intensity of the sound is increased with time in the form of a step function (Fig. 2). 


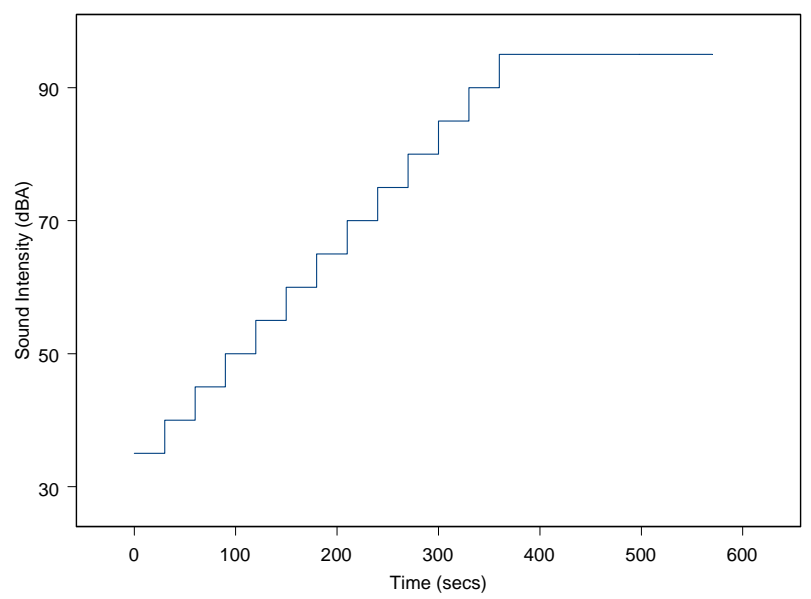

Fig.2. Time dependence of sound intensity.

The way the sound intensity increases with time is intended to ensure that all subjects eventually wake up, at least theoretically. This takes away one of the observational values used to fit the model, namely the probability of not awakening. On this account it is appropriate to modify the random walk model described above by removing the lower boundary. A single upper boundary model has been used by Smith [5]. It was also recommended to us by R.Ratcliff, one of the originators of the random walk model [6], in a private communication dated 29 January 2003. Removing the lower boundary leaves us with just three parameters: the wake up threshold $A$, the recognition probability $p$ and the length $d$ of a step.

There are in principle two ways of accounting for the increase in the sound intensity:

1. decreasing the step length. This is on the assumption that as the sound intensity increases the brain processes the incoming information faster;

2. decreasing the height of the threshold. This is on the assumption that as the sound intensity increases the sleeping subject becomes more sensitive to the matches.

There are good grounds to believe that the speed of information processing is not significantly affected by the intensity of the stimulus, so that in this paper the increase in sound intensity will be assumed to just lower the threshold and the step length will be kept constant.

It must be pointed out that the intensity of sound is actually measured in decibels. But the decibel is a logarithmic unit of sound intensity and according to the well-known WeberFechner law (see e.g., Encyclopaedia Britannica) human response to a stimulus is proportional to the logarithm of the stimulus. It follows that the response to a sound stimulus measured in decibels can be expected to vary linearly with the sound measurement. It was therefore decided to decrease the threshold linearly from some initial value $A$ at the points of time where the sound intensity was increased. The decrease 
was fixed at one unit. A graph showing the shape of the proposed threshold shape is given in Fig. 3.

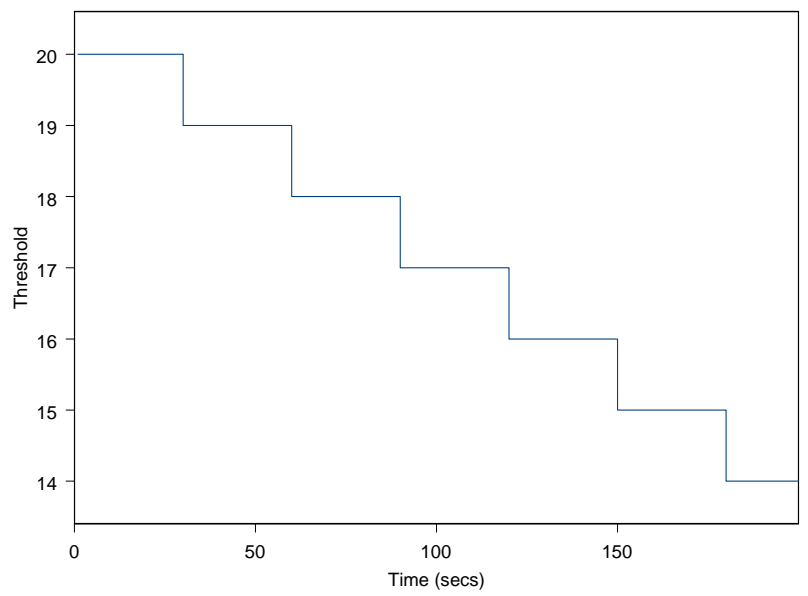

Fig. 3. Time dependence of threshold.

In addition, it must be pointed out that, as in the original random walk model described above, one of the parameters can be chosen arbitrarily. Changing that parameter simply changes the coarseness of the model. In the original random walk model described above it was $A$ that was chosen arbitrarily. In this paper we shall choose $d$ to be 0.5 seconds, and estimate $A$ and $p$ from the mean and standard deviation of the data.

\section{FITTING THE MODEL TO THE DATA}

In order to fit the model to the data it is necessary to be able to evaluate the mean and coefficient of variation of the time to reach the time dependent boundary (The first passage time) in terms of $A, p$ and $d$. Unlike the original random walk model described above this is by no means an elementary problem. In fact, we were unable to find a method to carry out the calculations in the literature. Eventually we developed our own algorithm. It is described in the Appendix. The first passage time probabilities are calculated by recurrence. It turned out that calculating 4000 successive first passage time probabilities were amply sufficient to obtain reliable values for the mean and the coefficient of variation.

The mean and coefficient of variation of the time to reach the boundary were calculated for an appropriate set of values of $A$ and $p$. The values of $A$ and $p$ corresponding to the observed values of the mean and standard deviation were then derived by interpolation. The fit is not entirely precise because of the fact that the threshold values are restricted to be integers.

A typical theoretical distribution function for the first passage time, as obtained from the formulae of the Appendix, is shown in Fig. 4.

It corresponds to the parameters $A=38, p=0.55$ and $d=0.5$ seconds, derived from the response to the T3 Alarm by sober males. The observed values are plotted together with the theoretical distribution. It can be seen that the fit is satisfactory. 


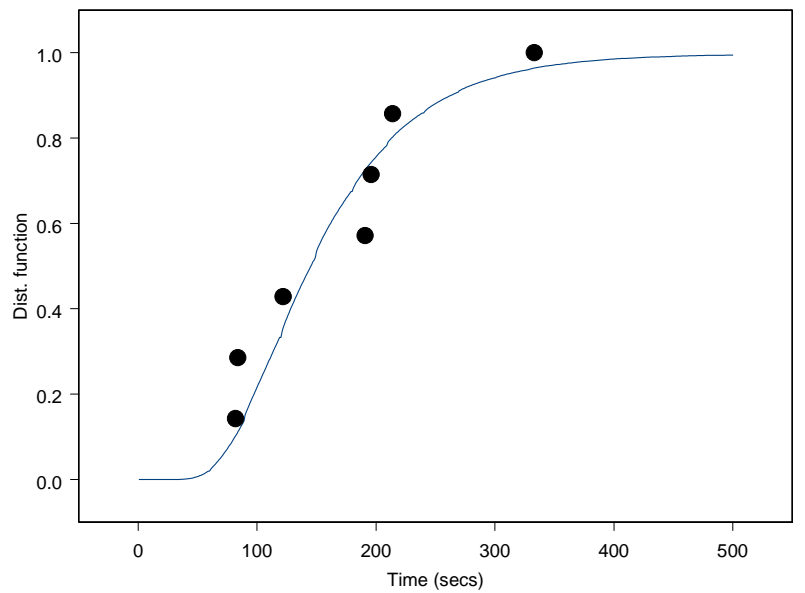

Fig. 4. Typical distribution function of first passage time.

(Corresponding to the response of sober males to the T3 Alarm with observed values of response time plotted for comparison).

\section{RESULTS}

The results of estimating $A$ and $p$ from the data are given in Table 2. While the threshold varies widely (between 33 and 62), the recognition probability is confined to a narrow interval just above 0.5 (between 0.52 and 0.55 ). This latter result is consistent with the analysis in [3], taking into account the fact that the increasing sound intensity can be expected to do away with the lower values of $p$.

\section{Linear Modelling of the Threshold}

The fitted threshold was regressed on alcohol level as a continuous variable and on two categorical factors: gender and type of sound. The regression on alcohol level was carried out as a polynomial regression of order two. The small amount of data did not justify a higher order of polynomial regression. Also, the fact that in addition approximations were made in the estimation process for the threshold and the recognition probability virtually vitiated the use of statistical tests of significance. For example, analysis of the raw data in [2 ] indicated that the female voice alarm and the T-3 sound were equally successful in all alcohol conditions, but that they were both significantly more successful than the Australian Standard Alarm Signal. This difference was lost in the estimation process for $A$ and $p$. It will undoubtedly reappear when more data are available. In the meantime this paper will concentrate on the gender and alcohol dependence of the parameters. 
Table 2. Threshold and recognition probability of the random walk model.

\begin{tabular}{|c|c|c|c|c|c|}
\hline Sound & Alcohol level & Male $\boldsymbol{A}$ & Female $\boldsymbol{A}$ & Male $\boldsymbol{p}$ & Female $\boldsymbol{p}$ \\
\hline Female & Sober & 37 & 33 & 0.55 & 0.55 \\
voice & 0,05 & 61 & 41 & 0.52 & 0.54 \\
& 0.08 & 57 & 43 & 0.53 & 0.54 \\
\hline ASA & Sober & 42 & 45 & 0.54 & 0.54 \\
& 0,05 & 58 & 45 & 0.53 & 0.54 \\
& 0.08 & 62 & 47 & 0.52 & 0.54 \\
\hline T3 & Sober & 38 & 33 & 0.55 & 0.55 \\
Alarm & 0,05 & 54 & 40 & 0.53 & 0.55 \\
& 0.08 & 62 & 40 & 0.52 & 0.55 \\
\hline
\end{tabular}

\section{Graphical Illustration of the Gender and Alcohol Dependence of the Threshold}

The dependence of the threshold on gender and alcohol is illustrated in Fig. 5, where the fitted values of the threshold are plotted against gender and alcohol. From the figure it can be inferred that the threshold for females is consistently lower than for males, indicating greater sensitivity/responsiveness. On the other hand, the curves depicting the dependence on alcohol are practically parallel, so that we can infer that males and females are affected similarly by alcohol, as far as the variation of the threshold with alcohol is concerned. The starting point (i.e., when sober) illustrates an increased responsiveness (lower threshold) for females - consistent with [3]. In addition, there is a pronounced downwards departure of the curves from linearity. This is in accordance with the Weber-Fechner law previously mentioned. Unfortunately, the fact that only three levels of alcohol were used, namely $0,0.05$ and 0.08 BAC, ruled out testing the logarithmic aspect of the law.

\section{Linear Modelling of the Recognition Probability}

As for the threshold, the estimated recognition probability was regressed on alcohol level as a continuous variable and on two categorical factors: gender and type of sound. The regression on alcohol level was carried out as a polynomial regression of order two.

\section{Graphical Illustration of the Gender and Alcohol Dependence of the Recognition Probability}

The dependence of the recognition probability on gender and alcohol is illustrated in Fig. 6, where the fitted values of the recognition probability are plotted against gender and alcohol. From the figure it can be inferred that the recognition probability for females is consistently higher than for males, indicating greater ability to match the stimulus with the memory set. It is interesting to note that the difference of recognition probability between males and females reported here is almost exactly the same as the difference reported in [3]. and On the other hand, the curves depicting the dependence on alcohol are practically parallel, so that we can infer that males and females are affected similarly by alcohol, as far as the variation of the recognition probability is concerned. For both males and females alcohol lowers the recognition probability, resulting in an increase of recognition time. In addition, there is a pronounced upwards departure of the curves from linearity. This is again in accordance with the Weber-Fechner law previously mentioned . It is interesting to note that the recognition probability curves are almost exactly a mirror image of the threshold curves. 


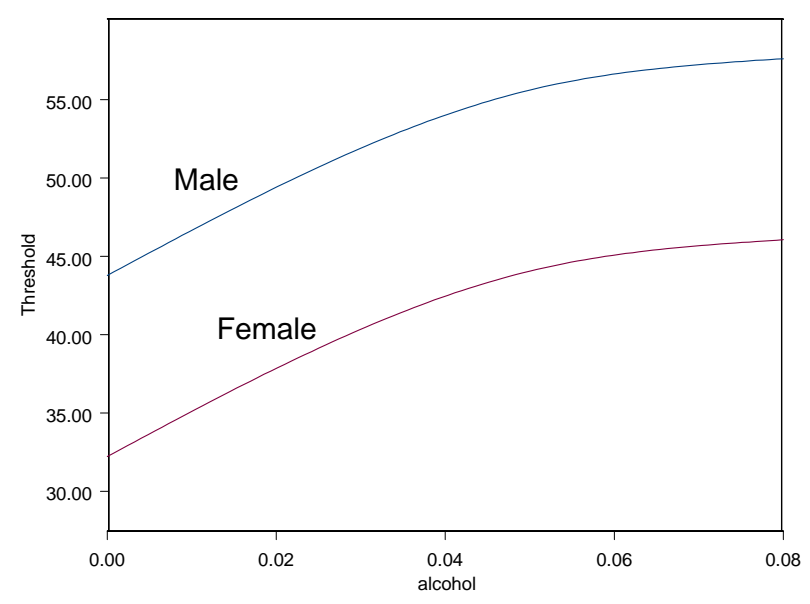

Fig. 5. Dependence of the threshold on gender and alcohol.

\section{THE ADVANTAGE OF USING THE PROPOSED MODEL}

As was pointed out in the Random Walk section, one of the most important aims of the stochastic analysis of the response time to fire cues is to estimate the high quantiles of the distribution of the response time, since it is those who respond the most slowly who are the most at risk. For the present model, all that is required is to evaluate the distribution function of the first passage time for values of $A$ and $p$ corresponding to the observed mean and standard deviation. From the distribution function it is easy to evaluate, say, the 99th percentile of the behavioural response time. For example, for the distribution function shown in Fig. 4, corresponding to $A=38$ and $p=0.55$, the 99th percentile of the response time turns out to be 435 seconds.

\section{SUMMARY}

The main conclusions of the paper are:

The response time by a sleeping subject to fire cues of increasing intensity may be modelled as a random walk with a decreasing upper boundary (threshold) and a fixed recognition probability.

Even with the very limited available data it was possible to establish that both threshold and recognition probability vary significantly with gender and alcohol consumption. Females have a lower threshold and a higher recognition probability than males. But both genders are significantly slowed down by alcohol, although the slowing down due to a 0.05 alcohol level is much higher than the subsequent slowing down with an increase from 0.05 to 0.08 alcohol level.

An effect detected by analysis of the raw data: that the ASA alarm was less effective than the female voice or the $\mathrm{T} 3$ alarm, was lost in the data manipulation.

Knowledge of the threshold and the recognition probability for a group of sleeping occupants allows the probability of long response times to be estimated. This is an essential parameter for safety management of fires. 


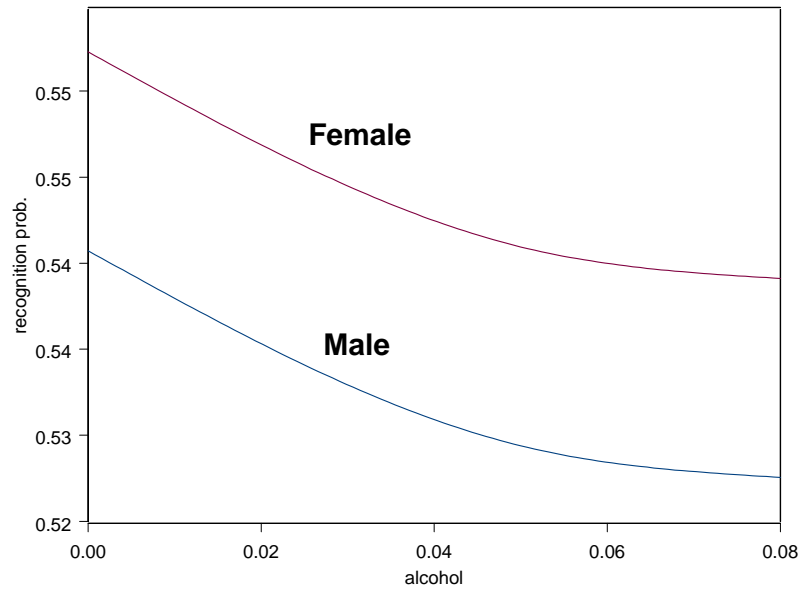

Fig. 6. Dependence of the recognition probability on gender and alcohol.

In this paper, the social impact of the results of the experiment, as far as the effect of alcohol and the type of alarm sound are concerned, are not discussed. For a comprehensive coverage of that topic, the reader is referred to the previously published paper of Ball and Bruck [2]. The purpose of the present paper is strictly to present a model that permits the estimation of the high quantiles of the response distribution, a core parameter in design for fire safety. It will of course be necessary to get far more data before the conclusions of the present experiment and its analysis can become fully established. 


\section{APPENDIX}

\section{Random walk with time-dependent absorbing boundary.}

We consider a random walk $W_{n}, n=0,1, \ldots$ starting at the origin (i.e. $W_{0}=0$ ), with $W_{n}=\sum_{i=1}^{n} Y_{i}, n=1, \ldots, \mathrm{P}\left(Y_{i}=+1\right)=p$ and $\mathrm{P}\left(Y_{i}=-1\right)=q,(p+q=1)$, and the $Y_{i}$ independent. The absorbing boundary is defined by the integer sequence $a=\left\{a_{n}\right\}, n=0,1, \ldots$ It is assumed that $a_{0}>0$ and $a_{1}>0$.

\section{Probability function of the unrestricted random walk}

Let $Z_{i}, i=1, \ldots$ be a sequence of independent Bernoulli variables, such that $\mathrm{P}\left(Z_{i}=\right.$ $1)=p$ and $\mathrm{P}\left(Z_{i}=0\right)=q$. Then the $Y_{i}$ of the previous paragraph have the same distribution as the variables $2 Z_{i}-1$, since $Z_{i}=1$ implies $2 Z_{i}-1=1$ and $Z_{i}=0$ implies $2 Z_{i}-1=-1$.

It follows that $W_{n}=2 \sum_{i=1}^{n} Z_{i}-n, n=1, \ldots$ Let $\sum_{i=1}^{n} Z_{i}=U_{n}$. Then $U_{n}$ is a binomial variable, with

$\mathrm{P}\left(U_{n}=k\right)= \begin{cases}\left(\begin{array}{l}n \\ k\end{array}\right) p^{k} q^{n-k} & \text { if } n \geq k \\ 0 & \text { otherwise. }\end{cases}$

Now, since $W_{n}=2 U_{n}-n$, it follows that

$\mathrm{P}\left(W_{n}=k\right)=\mathrm{P}\left(U_{n}=\frac{n+k}{2}\right)$.

But since $U_{n}$ must be an integer, we have

$\mathrm{P}\left(W_{n}=k\right)= \begin{cases}0 & \text { if } n+k \text { is odd } \\ \left(\begin{array}{l}n \\ r\end{array}\right) p^{r} q^{n-r} & \text { if } n+k=2 r \text { is even. }\end{cases}$

\section{Probability function of the first passage time}

Let $f_{m}$ be the probability that the random walk $W_{n}$ reaches the boundary $a$ for the first time at the $m$ th step.

Let $q_{m, n}$ be the probability that $W_{n}=a_{n}$, given that $W_{m}=a_{m}$ for some $m<n$. Let $p_{n-m, n}=q_{m, n}$ and write $p_{n}=q_{n, n}=p_{0, n}$ for short.

Consider the events $\mathrm{E}_{m}$ : "the random walk $W_{n}$ reaches $a$ for the first time at $m \leq n$ and $W_{n}=a_{n}$ ". The events $\mathrm{E}_{m}$ are mutually exclusive and $\mathrm{P}\left(\mathrm{E}_{m}\right)=\sum_{m} f_{m} q_{m, n}$. This can be written

$p_{n}=f_{n}+f_{n-1} q_{n-1, n}+f_{n-2} q_{n-2, n}+\cdots+f_{1} q_{1, n}$. 
or, equivalently,

$p_{n}=f_{n}+f_{n-1} p_{1, n}+f_{n-2} p_{2, n}+\cdots+f_{1} p_{n-1, n}$.

The argument leading to equation (5) is known as the renewal argument. (see Feller [7]). It has also been used by Durbin [8] to study the crossing of a time-dependent boundary by a diffusion process.

Since the $p_{m, n}$ can be calculated, using equation (3), the $f_{n}$ can be calculated by recurrence from equation (5) in the form

$f_{n}=p_{n}-\sum_{r=1}^{n-1} f_{r} p_{n-r, n}$.

\section{REFERENCES}

[1] Hasofer, A.M., "A Stochastic Model for the Time to Awaken in Response to a Fire Alarm,” J. of Fire Protection Engineering, 11, (3), pp. 151-160, (2001).

[2] Ball, M., and Bruck, D., "The Effect of Alcohol Upon Response to Different Fire Alarm Signals," Proceedings of the Third Human Behaviour in Fire Conference, Belfast, 1-3 October, London: Interscience Communications; 2004, pp. 291-301.

[3] Hasofer, A.M., and Bruck, D., "Statistical Analysis of Response to Fire Cues," Fire Safety Journal, 39, pp. 663-688, (2004).

[4] Proulx, G., and Laroche, C., "Recollection, Identification and Perceived Urgency of the Temporal-three Evacuation Signal,” Journal of Fire Protection Engineering, 13, pp. 67-82, (2003).

[5] Smith, P.L., "Psychophysically Principled Models of Visual Simple Reaction Time,” Psychological Review, 102, (3), pp. 567-593, (1995).

[6] Ratcliff, R., “A Theory of Memory Retrieval,” Psychological Review, 85, pp. 59-108, (1978).

[7] Feller, W., An Introduction to Probability Theory and its Applications, $3^{\text {rd }}$ Edition, Vol. I, Wiley, New York, (1968).

[8] Durbin, J., "Boundary-crossing Probabilities for the Brownian Motion and Poisson Processes and Techniques for Computing the Power of the Kolmogorov-Smirnov Test," Journal of Applied Probability, 8, pp. 431-453, (1971). 